\title{
Densely calcified tuberculous constrictive pericarditis with concurrent active pulmonary tuberculosis infection
}

\author{
Laudari $S^{1}$, Dhungel $S^{1}$, Gautam $S^{1}$,Dubey $L^{1}$, Prasad ${ }^{2}$, Bhattacharya $R^{2}$, Subramanyam $\mathbf{G}^{2}$
}

${ }^{1}$ DM Resident, ${ }^{2}$ Professor, Department of Cardiology, College of Medical Sciences, Bharatpur, Nepal

\section{ABSTRACT}

Though pulmonary tuberculosis is a common chronic infection in the developing countries like Nepal, the incidence of tubercular constrictive pericarditis is very low. Here we report a patient of active pulmonary tuberculosis with sputum positive for acid fast bacilli along with densely calcified constrictive pericarditis which has been reported as a very rare presentation in the literature.

Key words: Electrocariagraphy, Constrictive pericardditis, Tubercular, pericarditis.

\section{INTRODUCTION}

Constrictive pericarditis is a process of chronic fibrous thickening of the pericardium, which is frequently accompanied with calcification and prevents the diastolic filling of the heart.1,2 Many etiologies have been identified, such as idiopathic chronic pericarditis, infection, post-cardiac surgery, mediastinal radiotherapy, neoplastic, autoimmune, uremia, sarcoidosis and methysergide therapy.3,4

\section{CASE REPORT}

50 years old lady from a remote area (Humla) in Nepal presented with history of progressive swelling of lower limbs and abdomen for six months. She also had progressive shortness of breath with orthopnea and few episodes of bright red hemoptysis. Clinical examination reveals a lean and thin lady with muscle wasting, pallor, icterus, ascites, tender hepatomegaly and markedly elevated jugular venous

Correspondence: Dr. Shankar Laudari

Email:Ishankar2@hotmail.com descent. Kussmaul sign was positive. Pericardial knock was appreciated over the left sternal border during auscultation.

Electrocardiography showed nonspecific ST$\mathrm{T}$ wave abnormalities and reduced voltage. Chest X-ray was suggestive of very apparent dense circumferential pericardial calcification rim with right sided pleural effusion and left lower zone infiltrations as shown in Fig. 1(A). Pleural fluid tapping was done and it was straw coloured exudative fluid with positive adenosine deaminase (ADA) test. Cytology revealed chronic inflammatory cells with lymphocytes predominant. Sputum for acid fast bacilli was positive. CT scan thorax suggestive of dense pericardial calcification with pulmonary infiltrates and right sided pleural effusion as shown in Fig. 1(B and C). 2D Transthoracic echocardiography on four chamber apical view demonstrated diffuse pericardial calcification with notching on M-mode study as shown in Fig 2(A) and 2(B).It also revealed abrupt displacement of interventricular septum during early diastole, also known as septal "bounce"; marked respiratory variation in mitral, tricuspid inflow velocities and signs of systemic venous congestion such as dilation of hepatic veins and distention of the inferior vena cava 
Journal of College of Medical Sciences-Nepal, 2014, Vol-10, No-2

with blunted respiratory fluctuation and hepatic vein diastolic flow reversal during expiration. Tissue Doppler examination showed increased E2 velocity of the mitral annulus as well as septal abnormalities corresponding to the bounce.

Patient is advised for category-I anti-tubercular therapy and pericardiectomy as early as possible.

Figure 1.

Imaging studies of the patient 1 (A) a chest X-ray; 1(B) and 1(C) CT scan of the thorax showing thickened pericardium with dense areas of calcification, right sided pleural effusion and left lower zone infiltrations.

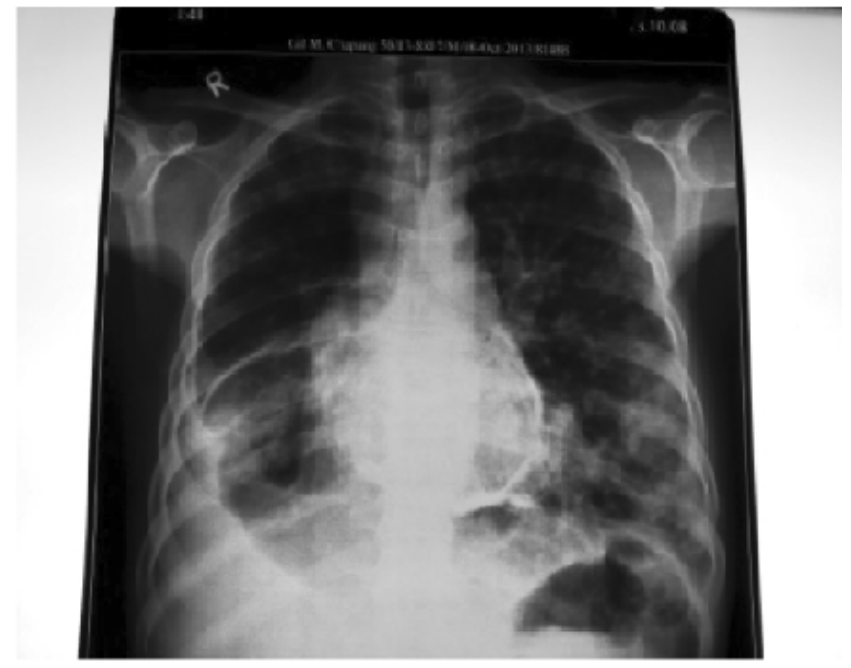

Fig. 1(A)

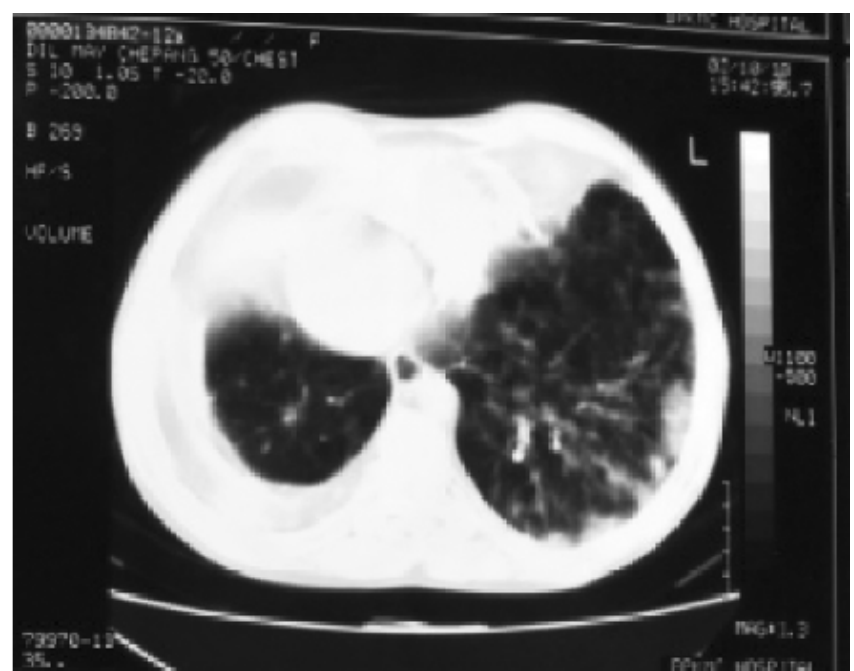

Fig. 1(B)

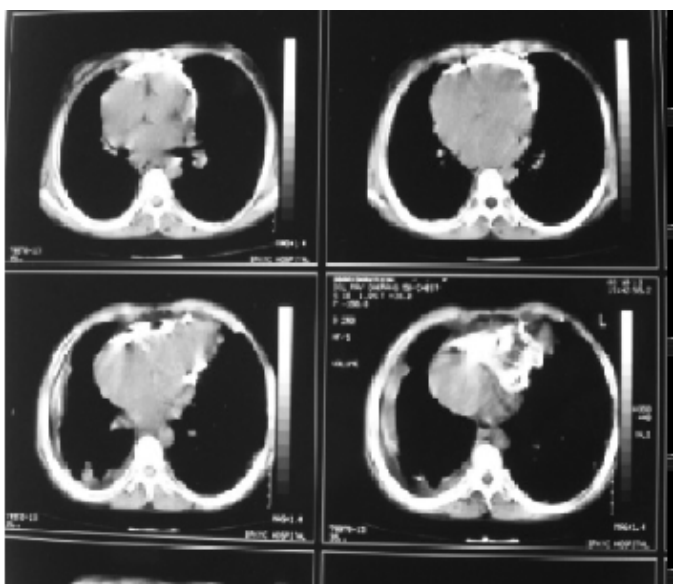

Fig. 1 (C)

Figure 2.

2D-Echocardiographic images study (A) Apical 4chamber view showing dense pericardial calcification (B) M-mode echocardiography showing diastolic notching of interventricular septum

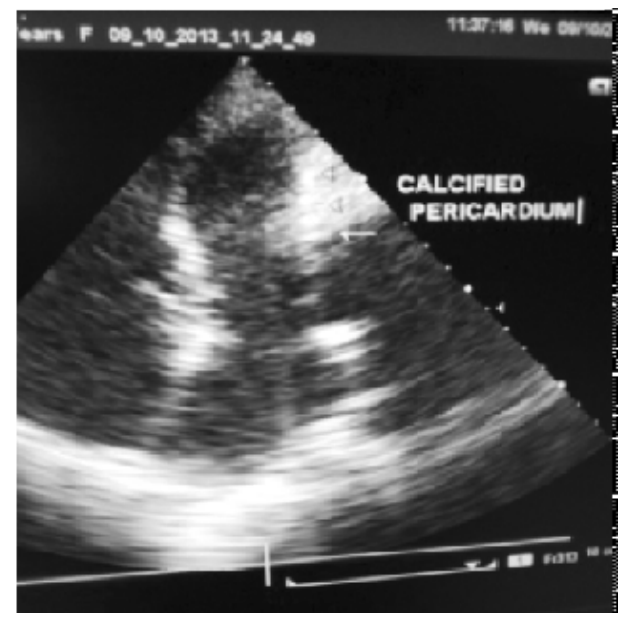

Fig. 2 (A)

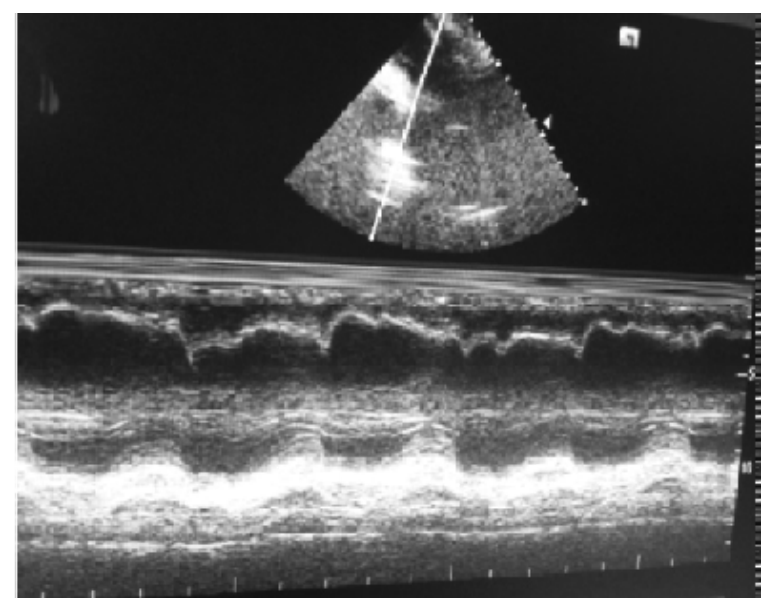

Fig. 2 (B) 


\section{DISCUSSION}

Tuberculous pericarditis will lead to pericardial constriction due to chronic granulomatous inflammation of the pericardium with subsequent deposition of fibrinous strands which is demonstrable on echocardiography. ${ }^{6}$

Anti-tuberculosis chemotherapy is still the cornerstone of the treatment for tuberculous pericarditis. In the endemic area, six-month anti-tuberculosis chemotherapy is recommended. In the chronic stage of tuberculous constrictive pericarditis, pericardial decortication with wide resection of both the visceral and the parietal pericardium, remains the definitive treatment. $^{2}$

Yang et al. highlighted the importance of pursuing early pericardiectomy, rather than pericardiocentesis and window placement, to achieve sustained relief of symptoms in patients with advanced stage disease. ${ }^{7}$

Tubercular constrictive pericarditis usually occurs much later after active tubercular pulmonary infection or ineffective therapy. There are very few reported cases of active pulmonary tuberculosis with concurrent constrictive pericarditis. Our case highlights the fact that tuberculosis is still a very common chronic infectious illness with very rare presentation. Strong suspicion should always be there for its early diagnosis so that effective therapy can be administered for better outcome.

\section{CONCLUSION}

Thorough clinical evaluation along with use of appropriate diagnostic tools and administration of definitive medical and surgical therapies are vital for better outcome of constrictive pericarditis. Surgical intervention still stands as the definitive therapeutic modality once the patient develops symptoms or signs of pericardial constriction and right sided heart failure.

\section{COMPETING INTERESTS}

The authors declare that they have no competing interests.

\section{REFERENCES}

1. Little WC, Freeman GL. Pericardial disease. Circulation. 2006;113:1622-32.

2. Maisch B, Seferovic PM, Ristic AD. Guidelines on the diagnosis and management of pericardial diseases: executive summary. Eur Heart J. 2004;25:587-610.

3. Bertog SC, Thambidorai SK, Parakh K. Constrictive pericarditis: etiology and cause-specific survival after pericardiectomy. J Am Coll Cardiol. 2004;44:1445-52.

4. Troughton RW, Asher CR, Klein AL. Pericarditis. Lancet. 2004; 363:717.

5. Chen R, Lai CP. Clinical characteristics and treatment of constrictive pericarditis in Taiwan. Circ J. 2005;69:458-60.

6. Liu PY, Li YH, Tsai WC. Usefulness of echocardiographic intrapericardial abnormalities in the diagnosis of tuberculosis pericardial effusion. Am J Cardiol. 2001;87:1133-35.

7. Yang CC, Lee MH, Liu JW. Diagnosis of tuberculous pericarditis and treatment without corticosteroids at a tertiary teaching hospital in Taiwan: a 14-year experience. J Microbiol Immunol Infect. 2005;38:47-52. 